\title{
Metas de inflação, taxa de câmbio real e crise externa num modelo kaleckiano
}

\author{
GABRIEL PORCILE \\ ALEXANDRE SOUZA \\ RICARDO VIANA*
}

\begin{abstract}
Inflation target, real exchange rate and external crisis in a Kaleckian model. Which role should the real exchange rate play in an inflation target regime? In this paper this point is discussed from the point of view of the conditions required for avoiding an external crisis. With this objective, a dynamic Kaleckian model is presented focusing on the stability of the external debt to capital ratio. The main conclusion is that policy makers should monitor closely the evolution of the real exchange rate in order to make compatible the inflation target regime with external stability.

Keywords: Kaleckian models; inflation target.

JEL Classification: F31; F34.
\end{abstract}

\section{INTRODUÇÃO}

Qual é o papel da taxa de câmbio real numa economia cuja regra de política monetária é a meta de inflação? Neste artigo o tema é discutido a partir da perspectiva das condições necessárias para a estabilidade de dívida externa e da possibilidade de emergência de crises no setor externo. Com esse objetivo desenvolve-se um modelo kaleckiano para uma pequena economia aberta cujo foco é a estabili-

\footnotetext{
* Respectivamente: Departamento de Economia da Universidade Federal do Paraná. E-mail: porcile@ ufpr.br (autor para correspondência); Banco Central do Brasil, Curitiba-PR. E-mail alexandrecgs@ gmail.; e Departamento de Física da Universidade Federal do Paraná, e-mail: viana@fisica.ufpr.br. As opiniões expressas neste trabalho são exclusivas dos autores e não refletem, necessariamente, as visões das instituições a que se vinculam. Os autores agradecem os comentários e sugestões de um parecerista anônimo, que foram extremamente úteis. Submetido: 18/5/2009; Aprovado: 3/9/2010.
} 
dade da relação dívida externa-capital. Já existe uma rica literatura pós-keynesiana sobre política monetária e instabilidade da dívida que serve de base para este trabalho, particularmente em torno da ideia de crises minskyanas ${ }^{1}$. Pretende-se fazer uma contribuição a essa literatura analisando as condições que propiciam o surgimento de instabilidade crescente numa economia na qual o governo segue um regime monetário exclusivamente baseado em metas de inflação.

A hipótese central a ser desenvolvida é que essa política pode conduzir a uma situação de instabilidade externa crescente se a evolução da taxa de câmbio real não for monitorada cuidadosamente pelos formuladores de política. Nesse sentido, o artigo sugere que, em certos casos, o fear of floating pode ser justificado. Em particular, políticas que colocam a competitividade do câmbio como elemento chave podem gerar trajetórias de crescimento mais estáveis que regras monetárias que atentam exclusivamente para o comportamento da inflação ${ }^{2}$.

O artigo está organizado em duas seções, além da introdução e das considerações finais. Na primeira seção apresenta-se o modelo kaleckiano simples de determinação do PIB como função das decisões de investimento e da taxa de câmbio real. Na segunda seção discutem-se as condições em que uma política de metas de inflação é consistente com uma relação dívida externa/PIB estável.

\section{UM MODELO KALECKIANO SIMPLES COM ENDIVIDAMENTO EXTERNO}

\section{O equilíbrio de curto prazo}

Assume-se uma economia aberta na qual não há política fiscal. O papel do governo é restrito à definição das metas de inflação e à administração da taxa de juros nominal em função dessas metas. Tomando-se como ponto de partida as identidades macroeconômicas básicas (1) e (2), e assumindo-se que trabalhadores não poupam, obtém-se a equação (3):

(1) $Y=C+I+B C$

(2) $Y=W+P$

(3) $C=W+(1-s) P, 0<s<1$

\footnotetext{
${ }^{1}$ Veja, por exemplo, Minsky (1975, 1986), Taylor e O’Connel (1985), Dymski e Pollin (1994), Foley (2003), Setterfield (2004a, 2004b), Meirelles e Lima (2006), Lima e Meirelles (2007). Devem ser incluídos também modelos pós-keynesianos voltados para problemas da gestão da política monetária, tais como Hein e Stockhammer (2007), Lavoie e Seccareccia (2004), Setterfield (2006) e Setterfield (Lima (2010). Uma discussão ampla dos avanços na macroeconomia de inspiração kaleckianakeynesiana encontra-se em Taylor (2004). Uma discussão com foco na América Latina encontra-se em Ocampo et al. (2009).

${ }^{2}$ Ver, por exemplo, Bresser-Pereira (2008), Basilio et al. (2008), Curado et al. (2008) e Gala (2008).
} 
$Y$ é o PIB, $C$ é o consumo agregado, $I$ é o investimento total, $B C$ o saldo de transações correntes (exportações líquidas), $P$ são os lucros, $W$ são os salários e $s$ é a taxa (exógena) de poupança. Todas as variáveis são definidas em termos reais.

A função de investimento é kaleckiana no sentido de que a taxa de crescimento do estoque de capital é uma função da diferença entre a taxa de lucro esperada $\left(r^{e}\right)$ e a taxa de juros real $\left(i_{r}\right)$, mais um componente autônomo $\left(g_{0}>0\right)$ que pode ser visto como refletindo o "animal spirit" keynesiano, ou mudanças estruturais e inovações exógenas ao modelo (Kalecki, 1997; Possas, 1987; Taylor e O’Connell, 1985). Não foram incluídos, no entanto, a taxa de utilização da capacidade instalada ou o papel das inovações, com o objetivo de obter um modelo mais simples que permita focar na dinâmica da dívida externa.

$$
\text { (4) } \frac{I}{K}=g=g_{0}+h\left(r^{e}-i_{r}\right), h>0
$$

As exportações líquidas reais são uma função do total de estoque de capital da economia $(K)$, da taxa de câmbio real (definida como $q=e p^{*} / p$, em que $e$ é a taxa de câmbio nominal, $p$ são os preços domésticos e $p^{*}$ os preços estrangeiros) e da propensão a importar $(m)^{3}$ :

(5) $B C=(a q-m) K, a \geq 0, m>0$

Baseado na equação de Fisher, a taxa de juros real pode ser escrita em termos da taxa de juros nominal $\left(i_{n}\right)$ e da taxa de inflação $(\pi)$ :

(6) $i_{r}=i_{n}-\pi$

As equações de (1) a (6) permitem encontrar a taxa de lucro de equilíbrio $r$ (em que $r=P / K$ ) e a taxa de equilíbrio da acumulação de capital $g$ (em que $g=I / K$ ) como funções da taxa de câmbio real e da taxa de juros nominais, em combinação com um conjunto exógeno de parâmetros positivos $\left(a, s, h, m, g_{0}\right)$.

(7) $r=\frac{g_{0}-h\left(i_{n}-\pi\right)+a q-m}{s-h}$

Rearranjando os termos em (7) e (4) resulta:

(8) $g=\frac{1}{s-h}\left\{g_{0} s+h\left[a q-m-\left(i_{n}-\pi\right) s\right]\right\}$

A equação (8) pode ser reescrita como uma curva IS kaleckiana:

(9) $g=A+B(a q-m)-C\left(i_{n}-\pi\right)$

Em que $A \equiv \frac{g_{o} s}{s-h}, B \equiv \frac{h}{s-h}$ e $C \equiv \frac{h s}{s-h}$.

\footnotetext{
${ }^{3}$ Para uma especificação similar, ver Basu (1984, 2003, p. 90), Blecker (2010) e Porcile e Lima (2010).
} 
A equação (9) representa o equilíbrio do mercado de bens, no sentido de que a demanda e a oferta agregadas são iguais (curva IS), como mostrado no Gráfico 1.

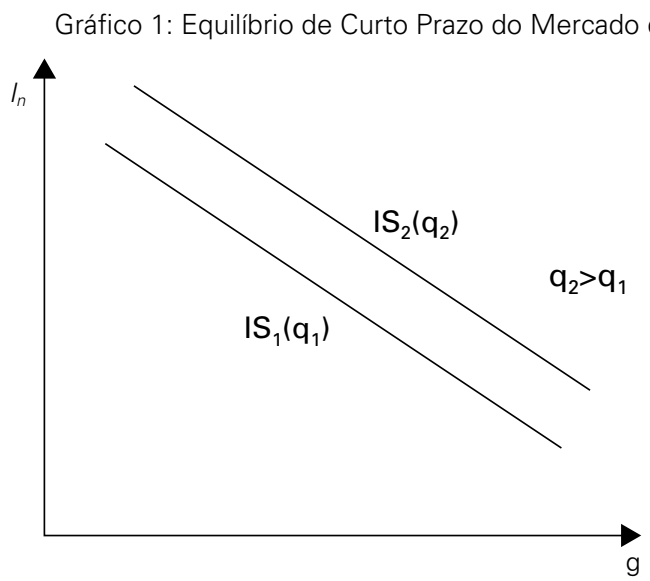

Ainda, esse é um equilíbrio de curto prazo que pode não ser sustentável dependendo da dinâmica do setor externo e da taxa de inflação. É necessário discutir como a taxa de câmbio real, a taxa de juros nominal e a taxa de inflação variam para produzir o equilíbrio de longo prazo, analisando a estabilidade desse equilíbrio. Este é o tópico para os próximos itens.

\section{Equilíbrio externo}

O equilíbrio externo requer não apenas equilíbrio no Balanço de Pagamentos, mas também a estabilidade da razão dívida externa por unidade de capital. Assumir-se-á que o débito externo é emitido no mercado internacional em moeda estrangeira à taxa nominal de juros internacional $i$ * mais um prêmio de risco $R$. A mudança no total do débito nominal (em moeda do país devedor) depende das exportações líquidas (eq. 5) e do pagamento de juros sobre o débito acumulado ${ }^{4}$ :

$$
\text { (10) } \frac{d(D p)}{d t}=-(a q-m) K p+\left(i^{*}+R\right)\left(D p^{*} e\right)
$$

Ao longo do trabalho será suposto que $R=0$, o que simplifica bastante a apresentação do modelo. Qualquer mudança na percepção do risco será analisada na forma de uma mudança exógena da taxa de juros internacional $i^{*}$. Tal suposto implica uma perda de riqueza da análise dinâmica, na medida em que se perdem não linearidades no comportamento da dívida e do crescimento, que surgem quan-

\footnotetext{
${ }^{4}$ Para uma especificação similar, veja a equação que baseia a teoria do ciclo da dívida de Simonsen e Cysne (2007, p. 90), $\dot{D}=i D-H$, em que $D$ é o passivo externo líquido, $i$, a taxa de juros incidente sobre a dívida pactuada em moeda estrangeira e $H$, a transferência líquida de recursos para o exterior, cujo valor é aproximado às exportações líquidas de bens e serviços do balanço de pagamentos.
} 
do se considera a taxa de risco uma variável endógena ${ }^{5}$. Em compensação, tal simplificação permite manter o foco no papel da taxa de câmbio real na preservação da estabilidade, que é a principal preocupação do trabalho. Mostrar-se-á que ainda no contexto mais favorável do ponto de vista da oferta de capitais (o nível da relação dívida - capital não altera a percepção de risco), caberá à variável câmbio um papel chave no sentido de garantir a estabilidade da economia.

Vale a penar notar que $d(D p) / d t=\dot{D} p+\dot{p} D$ e, portanto, $\frac{d(D p)}{p d t}=\dot{D}+D \pi$. A evolução do débito externo real $D$ (expresso em unidades do produto do país devedor) pode ser escrita como segue:

$\left(10^{\prime}\right)$

$$
\dot{D}=-(a q-m) K+D\left(i^{*} q-\pi\right)^{6}
$$

O débito real da razão do estoque de capital é $\delta=D / K$. A taxa de crescimento de $\delta$ é:

$$
\text { (11) } \hat{\delta}=\hat{D}-g
$$

Dividindo a equação (10') por D, e usando (11), obtém-se a equação dinâmica da razão débito real por capital (em termos do produto do país devedor):

(12) $\dot{\delta}=m-a q+\delta\left(i^{*} q-\pi-g\right)$

Lembrando que $g$ é uma função de $i_{n} \pi$ e $q$ (eq. 8), então a evolução de $\delta$ depende direta e indiretamente destas três variáveis. Uma maior taxa de inflação reduz a dívida externa em termos reais, enquanto um aumento na taxa de câmbio real aumenta o peso da dívida.

\section{Inflação e regra de Taylor}

É necessária uma equação para a taxa de inflação. A inflação responde a dois determinantes. Por um lado, ela depende da taxa de juros real $\left(i_{r}\right)$. Na tradição de conflicting claims, valores menores dessa taxa favorecem a expansão dos investimentos e do produto, elevando o emprego e as demandas salariais dos trabalhadores (que com mark up fixo redundam em aumentos de preços). Por outro lado, a inflação também depende da taxa de câmbio real. Parte da desvalorização da moeda nacional (e do aumento do custo dos bens de capital e insumos importados) é transferida aos preços (efeito pass-through), alimentando o processo inflacionário. Considerando a existência de uma relação unívoca entre taxas de juros nominal e real, pode-se escrever a taxa de inflação como uma função da taxa de juros nominal e da taxa de câmbio real, como na equação (13):

$$
\text { (13) } \pi=u q-v i_{n}
$$

\footnotetext{
${ }^{5}$ Por exemplo, Pereima Neto e Oreiro (2008) formalizam a percepção de risco como uma função da razão débito por capital, $R=\phi \delta$. Curado, Porcile e Viana (2007) utilizam uma taxa de juros crítica a partir da qual a entrada de capitais externos diminui.

${ }^{6}$ Por simplicidade, se assume que o termo de interação é pequeno.
} 
Os parâmetros $u$ e $v$ são positivos. Considerar-se-á que a taxa de câmbio real é dada no curto prazo e constante $(q-\tilde{q})$, já que ela de fato ajusta-se muito lentamente na direção do equilíbrio de longo prazo, no qual se verificam o princípio da paridade do poder de compra (PPP) e a condição de paridade não coberta de juros (UIP). Em um segundo momento, o artigo analisa o movimento da taxa de câmbio real na direção de seu equilíbrio de longo prazo, definido por aquele valor que satisfaz a igualdade das taxas reais de juros interna e externa (e que, portanto, atende PPC e UIP simultaneamente). O movimento da taxa de câmbio real na direção de seu equilíbrio de longo prazo ocorre através de sucessivos equilíbrios de curto prazo.

Finalmente, o governo determina a evolução dos juros nominais seguindo uma regra de Taylor simples, em que $\bar{\pi}$ é a meta de inflação a ser perseguida pelo Banco Central:

$$
\text { (14) } \frac{d i_{n}}{d t}=\alpha(\pi-\bar{\pi}) \alpha>0
$$

As equações (12) e (14) representam um sistema de duas equações diferenciais onde a relação dívida externa por capital e a taxa de juros nominal se definem endogenamente. $\mathrm{Na}$ seção seguinte, o sistema dinâmico formado por essas equações é estudado, focando nas condições que sustentam a estabilidade da dívida externa no tempo.

\section{METAS DE INFLAÇÃO E ESTABILIDADE DO SISTEMA}

\section{Equilíbrio e estabilidade}

Usando a equação (13) na equação (14), e considerando que a taxa de câmbio real no curto prazo é uma variável exógena $(\widetilde{q})$, obtém-se o seguinte sistema dinâmico com as variáveis de estado $i_{n}$ e $\delta$ :

(15) $\dot{\delta}=m=a \tilde{q}+\delta\left(i^{*} \tilde{q}-u \tilde{q}+v i_{n}-g\right)$

(16) $\frac{d i_{n}}{d t}=\alpha\left(u \tilde{q}-v i_{n}-\bar{\pi}\right)$

A matriz Jacobiana do sistema é, portanto:

$$
J=\left(\frac{\left(i^{*}-u\right) q+v i_{n}-g\left(\tilde{q}, i_{n}\right)}{0} \frac{\left[v-g^{\prime}\left(i_{n}\right)\right] \delta}{-\alpha v}\right)
$$

Lembrando que em equilíbrio $\bar{\pi}=u \tilde{q}-v i_{n}$, os sinais das derivadas parciais são os seguintes:

$\mathrm{J}_{11}=i^{*} q-\vec{\pi}-g$ : sinal indeterminado da resposta ao aumento da relação dívida / capital, dependendo da relação entre o pagamento de juros reais e a taxa de crescimento da economia. 
$\mathrm{J}_{12}>0$ : sinal positivo, porque $v$ e $\delta$ são positivos e $g^{\prime}\left(i_{n}\right)<0$. Juros maiores implicam menor investimento, aumentando a relação dívida / capital.

$\mathrm{J}_{21}=0$ : o Banco Central não inclui, na sua curva de reação, uma resposta ao aumento da relação dívida externa / estoque capital.

$\mathrm{J}_{22}<0$ : sinal negativo, porque $\alpha$ e $v$ são positivos. Juros mais altos tendem a reduzir a necessidade de aumento dos juros por parte do Banco Central, dada a meta de inflação.

Sendo assim, a condição de estabilidade dependerá basicamente do sinal de $\mathrm{J}_{11}$ e se resume à seguinte desigualdade:

$$
\mathrm{J}_{11}=\mathrm{CE}=i^{*} \tilde{q}-\bar{\pi}-g\left(\tilde{q}, i_{n}\right)<0
$$

CE é a condição de estabilidade. Caso se verifique que CE é negativa, então o traço da Jacobiana $\left(\mathrm{J}_{11}+\mathrm{J}_{22}\right)$ será negativo (pois $\left.\mathrm{J}_{22}<0\right)$ e o determinante $\left(\mathrm{J}_{11} \mathrm{~J}_{22}-\mathrm{J}_{21}\right.$ $\mathrm{J}_{12}$ ) positivo (pois $\mathrm{J}_{22}<0$ e $\mathrm{J}_{21} \mathrm{~J}_{12}=0$ ), sendo o sistema estável. Mas se CE for positivo, teremos um equilíbrio de ponto sela, que representa uma situação insustentável. Com efeito, a não ser no caso muito especial em que os valores iniciais se localizem no ramo estável do sistema, nem os juros nem a dívida permanecerão constantes, dando lugar a comportamentos explosivos. Esse tipo de comportamento é contraditório com o objetivo da política de manter a estabilidade macroeconômica; por essa razão o trabalho foca nas condições que garantem que $\mathrm{CE}<0$.

Os valores de equilíbrio de $i_{n}$ e $\delta$ são dados por:

$$
\text { (19) } i_{n}^{E}=\frac{u \tilde{q}-\bar{\pi}}{v}
$$

E o equilíbrio da taxa de crescimento é:

(20) $g^{E}=A-B m+C\left(1+\frac{1}{v}\right) \bar{\pi}+\left(B a-C \frac{u}{v}\right) \tilde{q}$

O Gráfico 2 ilustra o processo de ajuste para o equilíbrio. Tomando como condição inicial uma economia com taxas de juros muito altas (ponto $x$ ), tem-se que a taxa de juros nominal estará caindo, uma vez que a taxa de inflação é menor que a meta de inflação. Tanto a queda na taxa de juros como o aumento da inflação (que se move para cima, na direção da meta de inflação) reduzem a taxa real de juros, estimulando investimentos e, por conseguinte, a acumulação de capital. Taxas de juros mais baixas e maior crescimento levam a economia a um ponto no qual a razão dívida / capital começa a cair (atravessando a isóclina $\dot{\delta}=0$ ). Quando isto acontece, essa razão e a taxa de juros doméstica gradualmente evoluem em direção aos seus valores de equilíbrio7.

\footnotetext{
${ }^{7} \mathrm{~A}$ isóclina $\dot{\delta}=0$ é representada pela equação $\delta=(a q-m) /\left(i^{*} \tilde{q}-u \tilde{q}+v i_{n}-g\right)$. A derivada da isóclina com relação a $i_{n}$ é: $\partial(\delta) /\left.\partial i_{n}\right|_{\delta=0}=-(a \tilde{q}-m)\left(\left(v-g^{\prime}\left(i_{n}\right)\right) /\left[\left(i^{*}-u\right) \tilde{q}+v i_{n}-g\right]^{2}\right.$ Essa derivada será positiva se o sinal de
} 
Gráfico 2: O ajustamento ao equilíbrio de curto prazo

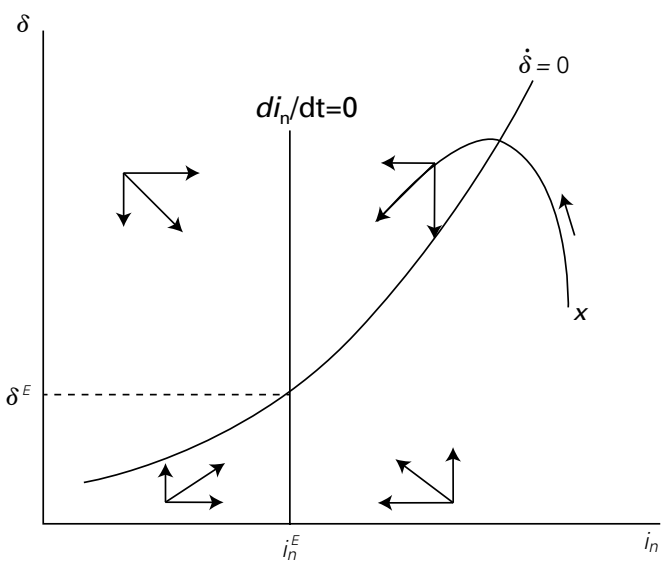

\section{O valor crítico da taxa de câmbio real}

Um aspecto interessante é que existe um limiar crítico para a taxa de câmbio real, abaixo do qual o sistema se torna instável. Usando a equação (17) e substituindo $g$ e $i_{n}$ nessa equação pelos valores de equilíbrio encontrados em (20) e (19), respectivamente, temos que:

$$
i^{*} \tilde{q}-\bar{\pi}-A-B(a \tilde{q}-m)+C\left(\frac{u \tilde{q}-\bar{\pi}(1+v)}{v}\right)<0 .
$$

Colocando $\tilde{q}$ em evidência, encontra-se finalmente que $\mathrm{CE}<0$ implica:

$$
\tilde{q}_{c}>\frac{-A v-\bar{\pi}[v+C(1+v)]+B m v}{v a B-C u-i^{*} v}
$$

A equação (21) será negativa para valores de câmbio real maiores do que $\tilde{q}_{c}$. Este valor da taxa de câmbio real representa um ponto de bifurcação: para valores abaixo desse ponto, a dinâmica do sistema é radicalmente alterada.

É importante ressaltar que há quatro cenários possíveis a depender do sinal do numerador e do denominador, analisados graficamente num apêndice no final do artigo. No gráfico 3 apresentamos apenas um desses cenários, no qual o numerador e o denominador de (21) são números positivos. Entende-se que esse é o cenário mais provável numa economia em desenvolvimento já que $m$ (a propensão a importar) é geralmente bastante alta, e as exportações tendem a ser mais sensíveis à taxa de câmbio real (na medida em que essa taxa compensa a mais baixa produtividade dessas economias, particularmente em setores de média tecnologia). Qual

$(m-a \tilde{q})\left(v-g^{\prime}\left(i_{n}\right)\right.$ for positivo. Como $v$ e $-g^{\prime}\left(i_{n}\right)$ são positivos, e os países em desenvolvimento apresentam em geral um déficit na balança comercial de forma que $(m-a \tilde{q})>0$, então a isóclina da razão débito por capital será positivamente inclinada no espaço $\left(\delta, i_{n}\right)$, como representado no gráfico 2 . 
seria o impacto, nesse contexto, de uma redução de $\tilde{q}$ ? Dado $\bar{\pi}=\bar{\pi}_{1}$, se a taxa de câmbio real cai para valores menores que $\tilde{q}_{c}$ o sistema se move da linha de estabilidade para a da instabilidade (ver Gráfico 3; notar que o coeficiente angular da linha $\bar{\pi}+g(\tilde{q})$ é maior do que o da linha $i^{*} \tilde{q}$, que implica $\left.(B a-C u / v)>i^{*}\right)$.

Gráfico 3: Taxa de câmbio e estabilidade da relação dívida / capital

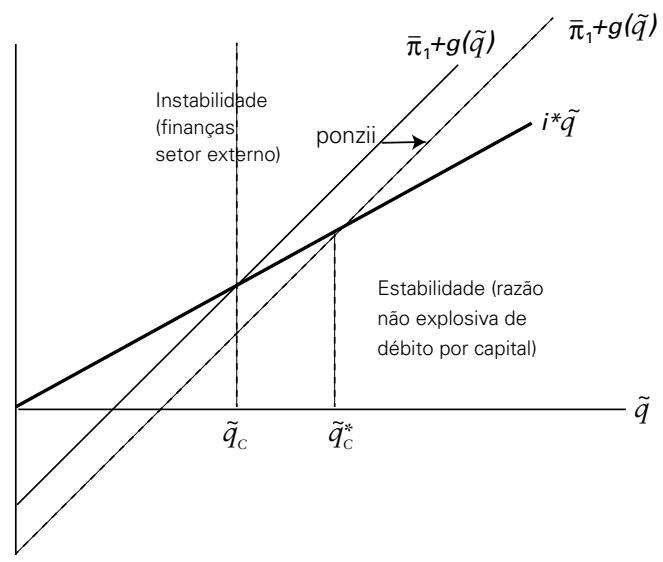

A influência da inflação definida como meta pode ser vista tomando a derivada de SC com respeito a $\bar{\pi}$, a qual é negativa:

$$
\frac{\partial\left[i^{*} \tilde{q}-\bar{\pi}-g\left(i_{n}(\bar{\pi})\right)\right]}{\partial \bar{\pi}}=-1-C\left(\frac{1+v}{v}\right)<0
$$

O Gráfico 3 mostra que a escolha de meta de inflação $\bar{\pi}$ mais baixa de $\bar{\pi}=\bar{\pi}_{1}$ para $\bar{\pi}=\bar{\pi}_{2}, \bar{\pi}_{1}>\bar{\pi}_{2}$ ) desloca a linha $\bar{\pi}+g(\tilde{q})$ para a direita (linha tracejada), fazendo necessária uma maior taxa de câmbio real para atingir o equilíbrio externo $\left(\tilde{q}_{c}^{*}\right)$. De fato, existe um trade-off entre $\bar{\pi}$ e $\tilde{q}$ pela qual estabilidade externa pode ser obtida com maior taxa de inflação e menor taxa de câmbio real, ou com maior taxa de câmbio real e menor taxa de inflação. Com efeito, permitindo tanto $\bar{\pi}$ e $\tilde{q}$ variar e tomando o diferencial total $\mathrm{d}(\mathrm{CE})=0$, obtém-se:

$$
d \tilde{q} / d \bar{\pi}=\frac{[v+C(1+v)]}{-C u-i^{*} v+v a B_{d C E=0}}<0
$$

A equação (23) explicita o trade-off entre a meta de inflação e a taxa de câmbio real compatível com a invariância da condição de estabilidade CE. Isto tem implicações interessantes do ponto de vista de política macroeconômica. Em alguns países, os governos têm mais capacidade de influenciar a meta de inflação do que a taxa de câmbio real. A última depende fortemente de variáveis estruturais relacionadas com a competitividade internacional ou com as condições institucionais de organização de negociação salarial no âmbito do mercado de trabalho, em razão do que é menos flexível. Os sindicatos resistem a taxas de câmbio real elevadas 
porque elas estão negativamente associadas com os salários reais (Carlin e Soskice, 1990; Blecker, 1998, 1999). Em uma economia em que sindicatos são muito fortes, de forma a definir um patamar baixo de taxa de câmbio real, ou baixa competitividade internacional, a estabilidade irá requerer maior taxa de inflação (dentro dos limites discutidos acima). De maneira oposta, se os sindicatos estão preocupados com a competitividade externa (portanto, com a taxa de emprego) e geram menor pressão sobre a taxa de câmbio real, a economia será capaz de atingir estabilidade externa com taxas mais baixas de inflação.

Um dilema mais complicado emerge quando ambos, sindicatos e Banco Central, tomam posições independentes dentro de suas próprias agendas, sem olhar para o contexto mais amplo da competitividade. Nesse caso, o resultado poderia ser um jogo de soma negativa levando às finanças Ponzi no setor externo. Tal situação aconteceria quando sindicatos restringissem a taxa de câmbio real definindo um valor baixo de $\tilde{q}$, enquanto o Banco Central insistisse em manter a inflação em níveis muito baixos. A combinação dessas duas forças reduz a razão débito externo / capital que será insustentável com o passar dos tempos.

A influência de mudanças endógenas nos parâmetros do modelo no valor de equilíbrio é apresentada no Quadro 1.

Quadro 1: Impacto na mudança do parâmetro sobre os valores de equilíbrio

\begin{tabular}{|c|c|c|}
\hline Variáveis/Parâmetros & & $(+)$ \\
\hline$i^{E}$ & $(-)$ & $(-)^{*}$ \\
\hline$\square^{\square}$ & $(-)$ & $(+)^{* *}$ \\
\hline$g^{E}$ & $(+)$ & \\
\hline
\end{tabular}

${ }^{*}$ Se $i^{*}<a(\pi+g) / m$

* *Se $\mathrm{B} a>\mathrm{Cu} / v$

O modelo sugere que metas de inflação e taxas de câmbio real relativamente altas favorecem o crescimento, mas obviamente esse resultado tem limites muito claros. Por um lado, se a taxa de inflação fosse muito elevada, ela poderia ficar fora de controle e gerar uma elevada instabilidade, algo que certamente esteve muito presente na história econômica brasileira e latino-americana (CEPAL, 2001; FFrench-Davis e Griffith-Jones, 1997; Ffrench-Davis, 1999; Frenkel e Taylor, 2006). Por outro lado, uma taxa de câmbio alta implica salário real deprimido. Há limites no uso da taxa de câmbio como fator de competitividade em sociedades democráticas. Caso se apostasse exclusivamente num câmbio alto, a situação poderia evoluir no sentido do que Fajnzylber (1990) chamava de competitividade espúria, baseada apenas em baixos salários e não em tecnologia mais avançada e produtividade.

Finalmente, o movimento da taxa de câmbio real na direção de seu equilíbrio de longo prazo é dado pela seguinte equação: 
(24) $\dot{q}=\tilde{\omega}(\bar{q}-q), \tilde{\omega}>0$

Qual é o valor de $\bar{q}$, a taxa de câmbio real de equilíbrio de longo prazo? Já foi mencionado que essa taxa satisfaz ao mesmo tempo PPC e UIP. Portanto:

(25) $i_{n}^{L P}-\bar{\pi}=i^{*}-\pi^{*}$

Em que $i_{n}^{L P}$ é a taxa de juros nominal de longo prazo. É fácil ver que:

(26) $i_{n}^{L P}=i^{*}-\pi^{*}+\bar{\pi}$

A essa taxa de juros de longo prazo corresponde uma taxa de câmbio real de longo prazo, que pode ser encontrada usando a equação (13):

(27) $\bar{q}=\frac{\left(\bar{\pi}+v i_{n}^{L P}\right)}{u}$

Usando a equação (26) em (27), após algumas simplificações, obtém-se:

(28) $\bar{q}=\frac{\bar{\pi}(1+v)+v\left(i^{*}-\pi^{*}\right)}{u}$

E finalmente, usando (28) em (24):

(29) $\dot{q}=\tilde{\omega}\left[\frac{\bar{\pi}(1+v)+v\left(i^{*}-\pi^{*}\right)}{u}-q\right]$

Com base na análise desenvolvida anteriormente com relação às condições de equilíbrio no setor externo, pode-se concluir que o sistema será estável no equilíbrio de longo prazo se $\bar{q}>\tilde{q}_{c}$, e além disso, se os valores assumidos por $q$ no seu trajeto para o equilíbrio também forem maiores do que $\tilde{q}_{c}$ (ou tiverem desvios muito pequenos que não violem a condição de equilíbrio antes discutida). Assim, o acompanhamento cuidadoso do comportamento da taxa de câmbio real, evitando a valorização da moeda local abaixo de certo patamar, seria uma condição-chave para que no longo prazo o regime de metas de inflação não gerasse problemas cumulativos de competitividade e desequilíbrio externo.

\section{CONSIDERAÇÕES FINAIS}

Neste ensaio foi apresentado um modelo de inspiração kaleckiana que procura identificar as condições capazes de produzir uma crise externa numa economia aberta em desenvolvimento na qual a política monetária tem como único objetivo atingir uma meta de inflação. A modelagem separa um curto prazo, em que a relação dívida por unidade de capital e taxa de inflação se equilibram, dada a taxa de câmbio real, e um longo prazo no qual a taxa de câmbio real evolui (lentamente) na direção do previsto pelo principio da paridade do poder de compra e pela paridade não coberta de juros. O ponto central do modelo é demonstrar que a 
taxa de câmbio pode induzir uma acumulação insustentável da dívida externa, se a taxa de câmbio real flutua para baixo até se tornar inferior a uma taxa de câmbio crítica. Em particular, uma meta de inflação reduzida e uma forte resistência sindical a uma queda do salário real (e, portanto, à elevação da taxa de câmbio real mais alta) podem resultar numa combinação particularmente explosiva. Essa combinação não tem sido rara em economias latino-americanas, onde crises externas foram recorrentes. Inversamente, países que têm implementado políticas focadas na manutenção dos níveis de competitividade internacional, como é o caso dos paises asiáticos, foram mais bem-sucedidos, tanto do ponto de vista do crescimento, como da estabilidade.

Alternativamente, o resultado do modelo pode ser lido da seguinte forma: uma política de metas de inflação pode ser adotada em economias abertas em desenvolvimento se ao mesmo tempo a taxa de câmbio real também entra como um objetivo de política macroeconômica, evitando sua redução em níveis que comprometam a estabilidade externa.

Sem dúvida, o modelo é bastante simplificado e deixa um conjunto muito grande de questões sem responder. Em particular, a política fiscal é uma grande ausente nessa discussão. Mas o modelo proposto deve ser entendido como um passo na direção da construção de modelos kaleckianos capazes de tratar analiticamente os temas da taxa de câmbio real em economias que combinam regimes de metas de inflação e problemas estruturais de baixa competitividade internacional.

\section{APÊNDICE: DISTINTOS CENÁRIOS DE DINÂMICA ECONÔMICA}

A condição de estabilidade em equilíbrio é dada pela desigualdade:

$$
\mathrm{CE}=i^{*} \tilde{q}-\bar{\pi}-A-B(a \tilde{q}-m)+C\left(\frac{u \tilde{q}-\bar{\pi}(1+v)}{v}\right)<0
$$

Isolando $\tilde{q}$, podemos dividir essa expressão em dois componentes:

A.1) $Z \equiv A+B m-\bar{\pi}\left[1+C\left(\frac{1+v}{v}\right)\right]$

A.2) $W \tilde{q} \equiv\left[i^{*}+C(u / v)-B a\right] \tilde{q}$

Assim, a condição de estabilidade pode ser escrita como:

A.3) $C E=Z+W \tilde{q}<0$

A.4) $\tilde{q}_{c}=-\frac{Z}{W}$

A partir daí podemos identificar distintos cenários em função dos valores dos parâmetros que definem $\mathrm{W}$ e $\mathrm{Z}$ e do valor crítico da taxa de câmbio real $\tilde{q}_{c}$. 
Caso 1: $\mathrm{Z}<0 \mathrm{e} \mathrm{W}>0$. $\mathrm{O}$ valor de $\tilde{q}_{c}$ é positivo: valores mais altos da taxa de câmbio real geram instabilidade e agravam o problema do setor externo (Gráfico 1.1).

Caso 2: $\mathrm{Z}<0 \mathrm{e} \mathrm{W}<0$. O valor de $\tilde{q}_{c}$ é negativo: para qualquer valor positivo da taxa de câmbio real o sistema é estável; não há problemas na frente externa (Gráfico 1.2).

Caso 3: $\mathrm{Z}>0 \mathrm{e} \mathrm{W}>0$. $\mathrm{O}$ valor de $\tilde{q}_{c}$ é negativo: para qualquer valor positivo da taxa de câmbio real o sistema é instável, e a taxa de câmbio real é incapaz de mudar isso (Gráfico 1.3).

Caso 4: $\mathrm{Z}>0$ e W $<0$. O valor de $\tilde{q}_{c}$ é positivo: a desvalorização da moeda contribui para a estabilidade do sistema e para o equilíbrio externo (gráfico $1.4 \mathrm{e}$ Gráfico 3 no texto).

Como se argumenta no texto, o Caso 4 é talvez o mais provável numa economia em desenvolvimento, já que nessas economias tendem a ser maiores a propensão a importar $(m)$ e a dependência das exportações com relação à taxa de câmbio real $(a)$.

Gráficos 1.1 - 1.4: Estabilidade e instabilidade do sistema dinâmico: distintos cenários

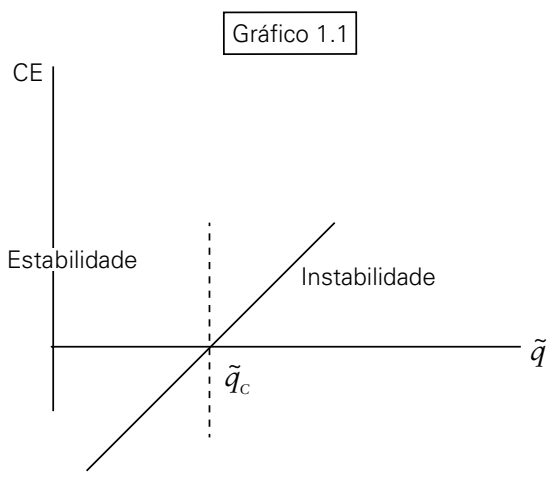

Gráfico 1.2

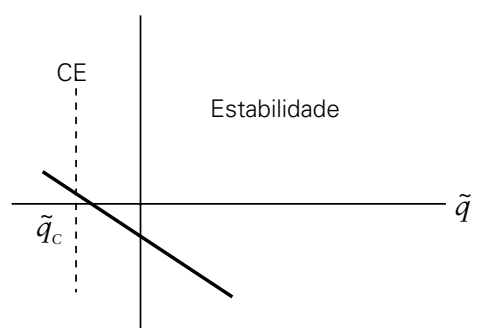

Gráfico 1.3

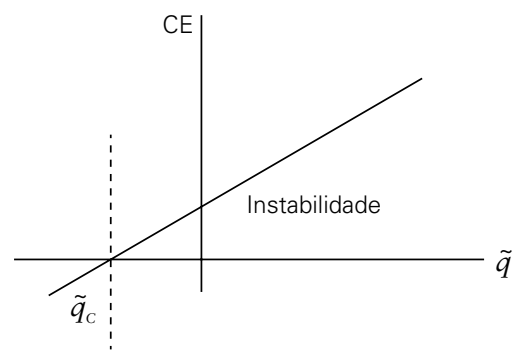

Gráfico 1.4

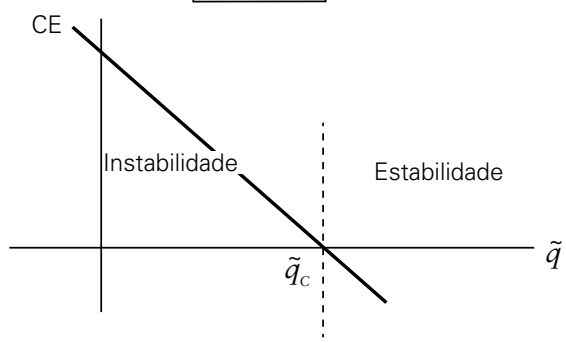




\section{REFERÊNCIAS BIBLIOGRÁFICAS}

BASILIO, A.C.; BASILIO, F; OREIRO, J.L. (2008) “Acumulação de Capital, Abertura Financeira e Endividamento Externo: um Modelo Macrodinâmico Pós-Keynesiano com Câmbio Flexível e Mobilidade de Capitais", Congresso Nacional de ANPEC, Salvador, 9-12 de dezembro.

BASU, K. (1984) The Less Developed Economy: A Critique of Contemporary Theory. Oxford: Basil Blackwell.

BASU, K. (2003) Analytical Development Economics: The Less Developed Economy Revisited. Cambridge, Mass.: The MIT Press, 2003.

BLECKER, R. (1998) "International competitiveness, relative wages and the balance-of-payments constraint", Journal of Post-Keynesian Economics, v. 20 (4), pp. 495-526.

BLECKER, R. (1999) "Kaleckian macro models for open economies," in Johan Deprez and John T. Harvey (ed.) Foundations of International Economics: Post Keynesian Perspectives. London: Routledge, pp. 116-149.

BLECKER, R. (2010) “Open economy models of distribution and growth", Paper presented at the Eastern Economic Association Annual Meeting, Philadelphia, PA, February 28, 2010.

BRESSER-PEREIRA, L.C. (2008) “The Dutch Disease and its neutralization: A Ricardian approach", Revista de Economia Política, Vol 28, n 1, Jan-Mar: pp. 47-71.

CARLIN, W., SOSKICE, D. (1990) Macroeconomics and the Wage Bargain. Oxford University Press.

CEPAL (2001) Una Década de Luces y Sombras: América Latina y el Caribe en los Años Noventa. Bogotá: Alfaomega.

CURADO, M.; ROCHA, M. e DAMIANI, D. (2008) "Taxa de câmbio e crescimento econômico: uma comparação entre economias emergentes e desenvolvidas", Congresso Nacional de ANPEC, Salvador, 9-12 de dezembro.

CURADO, M; PORCILE, G. e VIANA, R. (2007) “Technology, capital flows and the balance-of-payments constraint in a structuralist north-south model”, in Philip Arestis and Gennaro Zezza (eds), Advances in Monetary Policy and Macroeconomics, London: Palgrave.

DYMSKI, G. e POLLIN, R. (1994) New perspectives in Monetary Macroeconomics: Explorations in the tradition of Hyman P. Minsky. Ann Arbor: University of Michigan Press.

FFRENCH-DAVIS, R. e GRIFFITH-JONES, S. (1997) Os Fluxos Financeiros na América Latina: Um Desafio ao Progresso. São Paulo: Editora Paz e Terra.

FFRENCH-DAVIS, R. (1999) Macroeconomía, Comercio y Finanzas: Para Reformar las Reformas en América Latina. Santiago de Chile: McGraw Hill.

FRENKEL, R. e TAYLOR, L. (2006) "Real exchange rate, monetary policy and employment”, DESA Working Paper n. 19, Fevereiro.

FOLEY, D. (2003) "Financial fragility in developing economies", in Dutt, A. K. \& Ros, J. (eds) Development Economics and Structuralist Macroeconomics, Aldershot: Edward Elgar.

GALA, P. (2008) "Real exchange rate levels and economic development: theoretical analysis and econometric evidence”, Cambridge Journal of Economics, 32(2), pp. 273-288.

HEIN, E. e STOCKHAMMER, E. (2007) “A Post-Keynesian macroeconomic policy Mix as an alternative to the new consensus approach. Macroeconomic Policy Institute (IMK). Hans Boeckler Foundation. Working Paper 10/2007. Disponível em http://www.boeckler.de/pdf/p_imk_ wp_10_2007.pdf.

KALECKI, M. (1997) Teoria da Dinâmica Econômica: Ensaio sobre as Mudanças Cíclicas e a Longo Prazo da Economia Capitalista. São Paulo, Editora Nova Cultural.

LAVOIE, M. e SECCARECCIA, M. (2004) Central Banking in the MODERN WORLD: Alternative Perspectives. Cheltenham: Edward Elgar.

LIMA, G.T.; MEIRELLES, A. (2007) "Macrodynamics of debt regimes, financial instability and growth”. Cambridge Journal of Economics, v. 31(4), pp. 563-580.

LIMA, G.T. e SETTERFIELD, M. (2010) "Pricing Behaviour and the Cost-Push Channel of Monetary Policy, Review of Political Economy, 22 (1), pp. 19-40. 
MEIRELLES, A.; LIMA, G (2006). "Debt, financial fragility, and economic growth: a post Keynesian macromodel", Journal of Post Keynesian Economics, 29(1), pp. 93-115.

MINSKY, H. (1975) John Maynard Keynes. New York: Columbia University Press.

MINSKY, H. (1986) Stabilizing a Stable Economy. New Haven: Yale University Press.

OCAMPO, J.A.; RADA, C. and TAYLOR, L. (2009) Growth and Policy in Developing Countries: A Structuralist Approach. New York: Columbia University Press.

PEREIMA NETO, J. B. ; OREIRO, J.L. (2008) "Política fiscal, crescimento, distribuição de renda e regimes de endividamento público uma abordagem pós-Keynesiana”, Revista Análise Econômica, 49, Março: pp. 36-63.

PORCILE, G. e LIMA, G.T. (2010), "Real exchange rate and elasticity of labour supply in a balance-of-payments-constrained macrodynamics”, Cambridge Journal of Economics, (2010) 34(6): 1019-1039 first published online December 14, 2009 doi:10.1093/cje/bep065.

POSSAS, M.L. (1987) A Dinâmica da Economia Capitalista: Uma Abordagem Teórica. São Paulo, Editora Brasiliense.

SETTERFIELD, M. (2004a) "Financial fragility, effective demand and the business cycle". Review of Political Economy, 16 (2), pp. 207-223.

SETTERFIELD, M. (2004b) "Central banking, stability and macroeconomic outcomes: a comparison of new consensus and post-Keynesian monetary macroeconomics", in Lavoie, M.; Seccareccia, M. (2004) Central Banking in the Modern World: Alternative Perspectives. Cheltenham: Edward Elgar.

SETTERFIELD, M. (2006) "Is inflation targeting compatible with Post Keynesian economics?" Journal of Post Keynesian Economics, 28 (4), pp. 653-671.

SIMONSEN, M.H.; CYSNE, R. (2007). Macroeconomia. São Paulo, Atlas. $3^{a}$ Edição.

TAYLOR, L. (2004) Reconstructing Macroeconomics. Harvard University Press.

TAYLOR, L.; O’CONNELL (1985) “A Minsky crisis”. Quarterly Journal of Economics, 100, Supplement, pp. 871-885. 